\title{
Electron Impact Ionization of Lithium
}

\author{
S. M. Younger*† \\ National Bureau of Standards, Washington, DC 20234
}

August 18, 1981

\begin{abstract}
The electron impact ionization cross section of the neutral lithium atom has been calculated in a distorted wave exchange approximation. The total cross section is in good agreement with available experimental data at incident electron energies above $10 \mathrm{eV}$. Analytic fits are provided for the $1 s$ and $2 s$ subshell partial cross sections.
\end{abstract}

Key Words: Electron-atom scattering; electron impact ionization; lithium atom.

\section{Introduction}

Cross sections for the electron impact ionization of lithium atoms are required for studies of neutral beam injection in thermonuclear plasma machines. Experimental electron impact ionization cross sections for neutral lithium have been reported by Jalin et al. [1] for the incident electron energy range 100 to $2000 \mathrm{eV}$ and by Zapesochnyi and Aleksakhin [2] for the threshold to $30 \mathrm{eV}$ range. Earlier experiments include the relative measurements of Brink [3] and the absolute cross sections of McFarland and Kinney [4], both restricted to energies well above threshold. Theoretical calculations $[5,6,7]$, mainly in the plane wave $B_{c}$ m approximation, support the high energy data of Jalin et al. [1], which is more than a factor of two below the McFarland and Kinney [4] results.

The present work deseribes the application of the distorted wave exchange approximation to the calculation of the neutral lithium electron ionization cross section. This method, which has been described in detail in previous publications $[8,9$, 10], is an improvement over previous theoretical work on lithium in that: (1) it utilizes a more accurate ground state target wavefunction, (2) it allows for potential distortion effects for all three continuum electrons involved in the ionization process, (3) it considers the effect of scattering exchange, and (4) it includes inner shell ionization.

\section{Technique and Results}

The distorted wave exchange approximation used in the present work has been described in detail in previous publications [8-10]. The target was described by the HartreeFock ground state wavefunction given by Clementi and Roetti

* Center for Radiation Reseurch, National Measurement Laboratory.

†Supported in part by the Deparment of Energy, Office of Fusion Energy.
[11]. The ionization energies for the ejection of a $2 s$ and a $1 s$ electron were $I_{2 s}=5.393 \mathrm{eV}$ and $I_{1 S}=64.9 \mathrm{eV}$. The incident and final scattered partial waves in the direct matrix element were computed in the static local potential of the ground state plus a semiclassical energy dependent exchange potential. The ejected partial wave was computed in the potential of the ion with a $2 s$ or $1 s$ vacancy depending on whether outer or inner shell ionization was being considered. For the exchange matrix elements the final state partial wave potentials were reversed, and the phase of the matrix element was chosen so as to maximize the effect of exchange and hence minimize the total cross section [8]. The maximum partial wave orbital angular momenta considered were 14 for the incident and scattered waves and 10 for the ejected waves, sufficient to obtain convergence of the partial wave series.

The total cross sections for outer and inner shell ionization of $\mathrm{Li} \mathrm{I}$ are given in table $\mathrm{l}$.

Rather than calculate individual cross sections at high incident electron energy, which would be costly due to the large number of partial waves involved at high energies, we have used a fitting procedure [12] to obtain analytic expressions for the cross sections based on low energy distorted wave data and a Bethe slope derived from the photoionization calculations of McDowell and Chang [13] for the $2 s$ subshell

TABLE 1. Electron impact ionization cross sections for Lithium $\left(10^{-16} \mathrm{~cm}^{2}\right)$

\begin{tabular}{c|c|c|c|c|c}
\hline \multicolumn{3}{c}{ Is subshell } & \multicolumn{3}{c}{ 2s subshell } \\
\hline$u_{1}$, & $\begin{array}{c}\text { Ex- } \\
\text { change }\end{array}$ & $\begin{array}{c}\text { No-Ex- } \\
\text { change }\end{array}$ & $u_{2}$ & $\begin{array}{c}\text { Ex- } \\
\text { change }\end{array}$ & $\begin{array}{c}\text { No-ex- } \\
\text { change }\end{array}$ \\
\hline 1.25 & 0.0155 & 0.0184 & 1.25 & 2.95 & 2.50 \\
1.50 & .0292 & .0356 & 1.50 & 3.91 & 3.53 \\
2.25 & .0523 & .0632 & 2.25 & 4.51 & 4.35 \\
3.50 & .0627 & .0711 & 3.50 & 4.01 & 4.35 \\
4.00 & .0629 & .0729 & 4.00 & 3.76 & 4.16 \\
5.00 & .0611 & .0696 & 5.00 & 3.35 & 3.76 \\
\hline
\end{tabular}


and of Manson [14] for the $1 s$ subshell. The resulting expressions describing the electron impact ionization of lithium are

$$
\begin{aligned}
E Q_{2} & =251 .\left(1-\frac{1}{u_{2 s}}\right)-121 \cdot\left(1-\frac{1}{u_{2 t}}\right)^{2} \\
& +11.1 \ell_{n u_{2 t}}-\frac{157 .}{u_{2 s}} \ell_{n u_{2 t}}
\end{aligned}
$$

and

$$
\begin{aligned}
E Q_{1 s} & =26.9\left(1-\frac{1}{u_{1 s}}\right)-12.5\left(1-\frac{1}{u_{1 s}}\right)^{2} \\
& +10.7 \ell n u_{1\lrcorner}-\frac{33.7}{u_{1 s}} \ell_{n u_{1 s}}
\end{aligned}
$$

where $u_{14}=E / I_{1 s}$ and $u_{2}=E / I_{2}$ with the incident electron energy, $E$, in eV. $Q$ is the cross section in $10^{-16} \mathrm{~cm}^{2}$.

Figure 1 compares the present results with the available experimental data and other theoretical calculations. The distorted wave data are in good agreement with the more recent experiments, except for incident electron energies below about
$10 \mathrm{eV}\left(u_{2}<1.85\right)$ where a more complex interaction between the target and scattering wave is present. The Born approximation calculations of McGuire [5] and of Peach [6] are 1050 percent lower than the present results, and are in slightly better agreement with experiments at very low and very high incident electron energies. McGuire's calculation includes the contribution from $1 s$ ionization; Peach's results are for $2 s$ ionization only.

The distorted wave method is essentially a model potential scattering theory which assumes that the target atomic structure is unaffected by the presence of the scattered electrons. While numerous attempts have been made to include polarization effects, electron correlation and even relativistic corrections in a distorted wave model, it is not clear to what extent such modifications are true improvements in such a simple theory versus attempts to obtain a better fit to numerical data. The present choice of a direct local potential with a semiclassical exchange potential has been found to yield partial wave phase shifts in remarkable agreement with frozen core Hartree-Fock calculations, and to produce reasonably accurate total ionization cross sections for a wide variety of atoms and ions $[8-10,12]$. Attempts to improve the distorted wave exchange ionization theory, specifically by

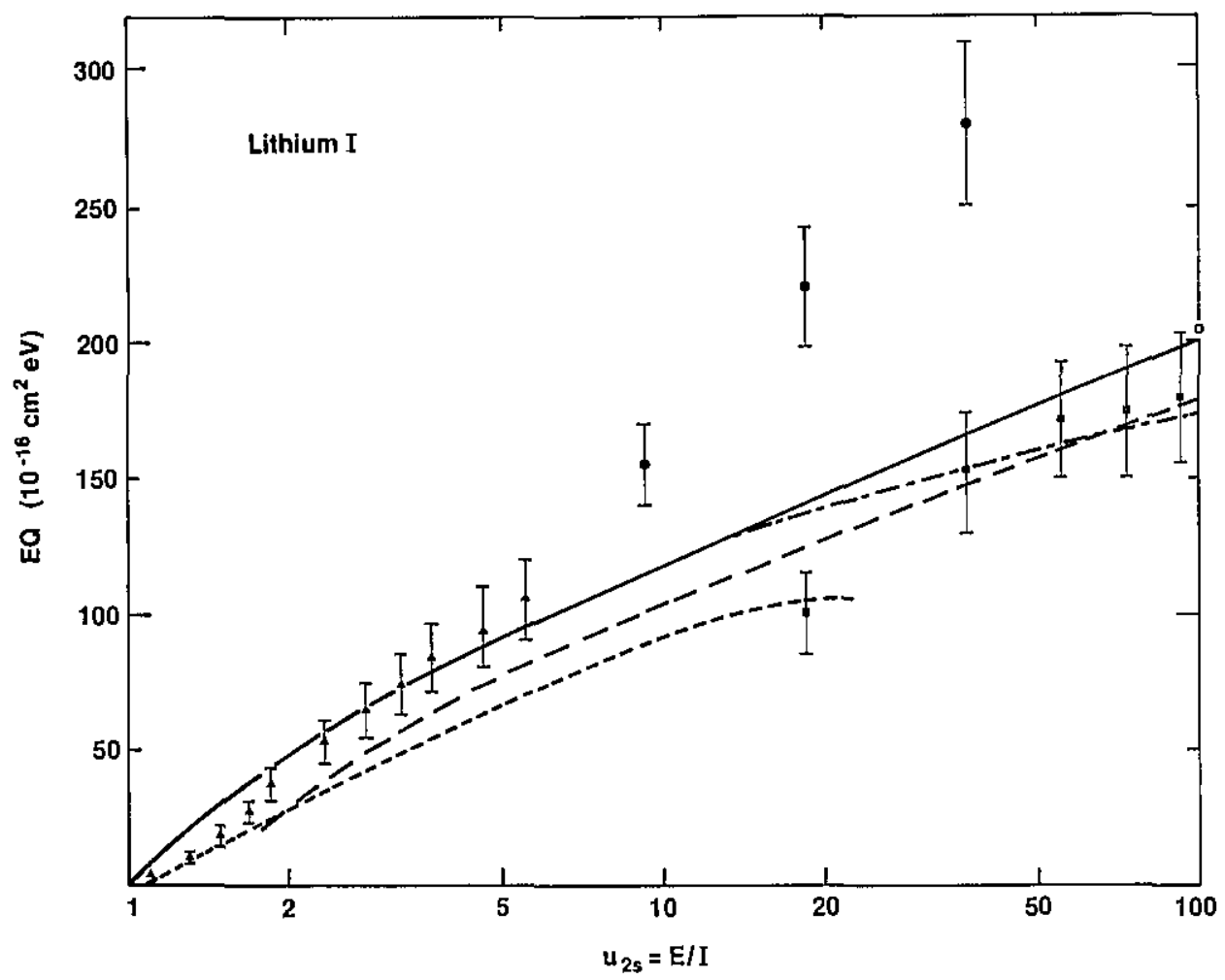

FIGURE 1. Fano plot of the electron impact ionization cross section of lithium. — total ionization cross section computed in the distorted wave exchange approximation (present); $-\cdot-\cdot-2 s$ ionization cross section, distorted wave exchange approximation (present); _—_-Plane wave Born, Ref. [5]; ...-.Plane wave Born, Ref. 6; 0 Bethe theory, Ref. 7; Crossed-beam experiment, Ref. [4]; $\triangle$ Crossed-beam experiment, Ref. 2; Crossed-beam experiment, Ref. [1] 
means of polarization potentials, have met with mixed success, improving agreement with experiment in some cases and causing greater disagreement in others. The present results are felt to represent a realistic compromise between the simplicity of the plane wave Bom approximation and the rigors of a full many body approach.

\section{References}

[1] Jalin, R., Hagemann, R., and Botter, R., J. Chem. Phys. 59, 952 (1973).

[2] Zapesochyni, I.P., and Aleksakhin, I.S., Zh. Eksp. Teor. Fiz 55, 76 (1968) [JETP 28, 41 (1969)].
[3] Brink, G.0., Phys. Rev. 134, A345 (1964).

[4] McFarland, R.H., and Kinney, J.R., Phys. Rev. 137, 1058 (1965).

[5] McGuire, E.J., Phys. Rev. A 3, 267 (1971).

[6] Peach, G., Proc. Phys. Soc. 87, 381 (1966).

[7] Kim, Y.X., and Inokuti, M., Phys. Rev. A 3, 665 (1971).

[8] Younger, S.M., Phys. Rev. A 22, 111 (1980).

[9] Younger, S.M., Phys. Rev. A 22, 1425 (1980).

[10] Younger, S.M., Phys. Rev. A 23, 1138 (1981).

[11] Clementi, E., and Roetti, C., At. Data Nuc. Data Tables 14, 177 (1974).

[12] Younger, S.M., J. Quant. Spectrosc. Rad. Transfer, (1981).

[13] McDowell, M.R.C., and Chang, E.S., Mon. Not. R. Astron. Soc. 142, 465 (1969).

[14] Manson, S., private communication. 